\title{
Relative Quantitation of Neuropeptides over a Thousand-fold Concentration Range
}

\author{
Xiaowen Hou, ${ }^{1,2}$ Fang Xie, ${ }^{2,3,4}$ Jonathan V. Sweedler ${ }^{2,3}$ \\ ${ }^{1}$ Center for Biophysics and Computational Biology, University of Illinois at Urbana-Champaign, Urbana, IL, USA \\ ${ }^{2}$ The Beckman Institute, University of Illinois at Urbana-Champaign, Urbana, IL, USA \\ ${ }^{3}$ Department of Chemistry, University of Illinois at Urbana-Champaign, Urbana, IL, USA \\ ${ }^{4}$ Pacific Northwest National Laboratory, Richland, WA 99352, USA
}

\begin{abstract}
Neuropeptides are essential cell-to-cell signaling molecules that influence diverse regulatory and behavioral functions within biological systems. Differing in their amino acid sequences and posttranslational modifications, hundreds of neuropeptides are produced via a series of enzymatic processing steps, and their levels vary with location, time, and physiological condition. Due to their wide range of endogenous concentrations and inherent chemical complexity, using mass spectrometry (MS) to accurately quantify changes in peptide levels can be challenging. Here we evaluate three different MS systems for their ability to accurately measure neuropeptide levels: capillary liquid chromatography-electrospray ionization-ion trap (CapLC-ESI-IT) MS, ultraperformance liquid chromatography-electrospray ionization-quadrupole-time-of-flight (UPLC-LC-ESI-QTOF) MS, and matrix-assisted laser desorption/ionization-time-of-flight (MALDI-TOF) MS. Specifically, eight sample mixtures composed of five neuropeptide standards, with four technical replicates of each, were labeled with $\mathrm{H}_{4} / \mathrm{D}_{4}$-succinic anhydride, followed by relative peptide quantitation using the three MS platforms. For these samples, the CapLC-ESI-IT MS platform offered the most robust ability to accurately quantify peptides over a concentration range of 1200 -fold, although it required larger sample sizes than the other two platforms. Both the UPLC-ESI-Q-TOF MS and the MALDITOF MS systems had lower limits of quantification, with the MALDI-TOF having the lowest. By implementing several data acquisition schemes and optimizing the data analysis approaches, we were able to accurately quantify peptides over a three orders of magnitude concentration range using either the UPLC or MALDI-TOF platforms. Overall these results increase our understanding of both the capabilities and limits of using MS-based approaches to measure peptides.
\end{abstract}

Key words: Quantitation, Neuropeptides, Liquid chromatography, Mass spectrometry, Peptidomics, Isotopic labeling, Large dynamic range

\section{Introduction}

$\mathrm{N}$ europeptides are signaling molecules that transmit information from cell to cell, modulating a variety of

\footnotetext{
Electronic supplementary material The online version of this article (doi:10.1007/s13361-012-0481-0) contains supplementary material, which is available to authorized users.
}

Correspondence to: Jonathan V. Sweedler; e-mail: jsweedle@illinois.edu activities within the nervous system that influence an organism's function and behavior. They are initially expressed as prohormones, which undergo a large number of enzymatic processing steps in the cells to produce the final bioactive peptides [1-3]. The functional effects of specific neuropeptides are dependent on their final chemical forms as determined by the particular enzymes present in the tissue and the order in which the enzymes process the prohormones. A large number of neuropeptides-differing in their amino acid sequences and post-translational mod- 
ifications (PTMs) - are produced within distinct brain regions. Furthermore, the peptidases responsible for prohormone processing may be differentially expressed in tissues [4-6] or during development [7], resulting in peptides that are differentially expressed, modified, cycled, and distributed. Thus, the specific forms and quantities of neuropeptides vary by region, upon release, and/or among developmental stages, and their dynamic range spans many orders of magnitude. These factors, combined with neuropeptides' inherent low abundance and the complexity of the surrounding biological matrices, present a variety of analytical challenges.

Accordingly, a number of technologies have been tailored to enable peptide discovery, essentially launching the field of study known as neuropeptidomics [3, 8-10], which endeavors to characterize the peptides present $[3,11]$ or released [12-14] in the nervous system. Neuropeptidome studies may also involve quantitation of neuropeptide level changes arising from different behavioral states, gene knockouts, or exposure to chemicals or drugs [15-21]. These examples involve tissue samples and represent the range of neuropeptide applications that have emerged. MSbased technologies have proven to be quite effective for peptide quantitation, with perhaps the two most common strategies being categorized as label-free [18, 22-25] and isotopic labeling [15-17, 20, 21]. Promising alternatives include a variant on the label-free method, termed spectral counting, which counts the number of times specific peptides are detected [26-29], the tried and true standard addition approach [20,30-33], and MS combined with microfluidics to probe the length of a channel that has peptides bound on it $[34,35]$.

Most label-free methods rely on the linear relationship between the intensity of the MS signal and the amount of analyte [36] in order to directly compare analyte levels. An important advantage is that these methods typically require fewer sample handling steps, which generally results in less sample loss. Therefore, label-free quantitation tends to work well for small-volume samples and low-abundance peptides. Another benefit is that these methods result in less complex samples compared with labeling strategies. There is also less chance of matrix interference when labeling chemicals are not used; this, combined with reduced sample losses, suggests that label-free methods may produce a higher coverage of peptides [37]. In addition, each sample is analyzed in parallel, so theoretically there is no limit to the number of samples that can be used for these types of MS comparison studies. However, there are caveats: because label-free approaches compare peptide levels from different MS analyses, there is a potential for larger systematic errors and poorer quantitation accuracy as compared to labeling methods [36, 37].

In isotopic labeling, the samples are differentially labeled and combined together, and then analyzed in a single MS or LC-MS analysis in order to compare the differences in peptide levels. This eliminates the analysis-to-analysis discrepancies that can result when using label-free strategies. As a minor point, isotopic labeling for peptidomic studies creates multiple tagged molecules for each peptide (distinguishable via their mass differences in MS or tandem MS modes) $[3,11,36]$. Although this makes the samples more complex, overall, isotopic labeling tends to provide a higher accuracy of quantitation than label-free approaches [37].

Numerous peptide quantitation studies have relied on isotopic labeling and MS [15-17, 20, 21, 38-47]. The labeling reagents most commonly employed for peptide quantitation of biological samples are the $\mathrm{H}$ - and D-forms of acetic anhydride [41, 44], succinic anhydride (SA) [15, 20, $39,41]$, and 4-trimethylammoniumbutyryl labels [16, 40]. These reagents react with the primary amines to label the free N-termini of the peptides, as well as the side chains of any internal lysine residues, and generate differentially labeled peptides having a mass difference of several Da, which in turn allows for differentiation and comparison. Other labels (e.g., urea and formaldehyde) have also been developed and tested using peptide/protein standards [4650]. Validation studies have evaluated the ability of isotopic labeling and MS to accurately quantify a single peptide standard or digests from a single protein over a concentration range of up to two orders of magnitude [43, 46, 47, 49]. In other studies, mixtures of peptide standards, with each peptide having the same concentration, were labeled differentially and combined in a 1:1 molar ratio [45] or ratios ranging from $4: 1$ to $1: 4$ [44]; these peptide mixtures were then quantitated via MS and the accuracy validated.

The examples cited above are well-performed validation studies, but typically used mixtures that did not mimic the large dynamic range of endogenous peptides that are present in a biological sample. Does this mean more studies are needed? Do these labeling schemes provide accurate quantitative results in samples containing peptides in amounts that span three or more orders of magnitude? Are there specific MS platforms better suited to investigate such real-world samples? If so, which data acquisition and analysis approaches work best?

In an effort to answer these questions, we compared the performance of three MS platforms that have been previously employed for peptide quantitation - capillary liquid chromatography-electrospray ionization-ion trap (CapLCESI-IT) MS [43], ultraperformance liquid chromatographyelectrospray ionization-quadrupole-time-of-flight (UPLCLC-ESI-Q-TOF) MS [15], and matrix-assisted laser desorption / ionization-time-of-flight (MALDI-TOF) MS [20]. We quantified a number of isotopically labeled neuropeptides, with ratios ranging from 12:1 to $1: 12$, in mixtures with peptide concentrations ranging over three orders of magnitude (within each mixture). We selected $\mathrm{SA}\left(\mathrm{H}_{4}\right.$ and $\mathrm{D}_{4}$ forms) as the labeling reagent because of its high efficiency and stability throughout the sample preparation and measurement processes on all MS platforms used. In addition, the $4 \mathrm{Da}$ difference that exists between the H/D labels allowed us to distinguish the isotopologue clusters of the labeled and unlabeled forms of the peptides, and the coelution of differentially labeled peptides from reversed-phase 
columns [11]. Although we expect that the other labels would generate similar results, we did not confirm this.

Our results demonstrate that among the three platforms used here, CapLC-ESI-IT MS is the most robust option for quantifying peptides accurately over a wide concentration range. However, by optimizing the data acquisition to ensure that the peptide signals reach a threshold, and other adjustments, the quality of the results using the other two systems can be appreciably increased, allowing us to obtain the same dynamic range of performance as with the CapLC-ESI-IT MS system. Parameters other than dynamic range can also be considered when evaluating performance; for example, with MALDI MS we were able to quantify the peptide ratios at lower concentrations compared with the other platforms tested.

\section{Experimental}

\section{Chemicals}

Chemicals were obtained from Sigma-Aldrich (St. Louis, MO, USA) unless otherwise stated. The peptide standards for MALDI-TOF calibration were from Bruker Daltonics (Billerica, MA, USA). Milli-Q water was prepared with a Milli-Q 119 filtration system (Millipore, Bedford, MA, USA). HPLC-grade solvents were purchased from Thermo Fisher (Fair Lawn, NJ, USA).

\section{Sample Preparation and Isotopic Labeling}

Five neuropeptide standards (bradykinin1-7, NPSSFVRIamide, bradykinin, angiotensin I, neurotensin), as shown in Table 1, were individually dissolved to concentrations varying from 1 to $100 \mu \mathrm{M}$. The standards were mixed together to generate eight different peptide mixtures of each of the five peptides at various concentrations and ratios.

The eight peptide mixtures were divided into two sets of four, and each set was labeled with either $4 \mathrm{MH}_{4}$-(light) or $4 \mathrm{M} \mathrm{D}_{4}$-(heavy) $\mathrm{SA}$ dissolved in dimethyl sulfoxide (DMSO), after the $\mathrm{pH}$ of each set was adjusted to $\sim 9$ using phosphate buffer $(1 \mathrm{M}, \mathrm{pH}=9.5)$, or $1 \mathrm{M} \mathrm{NaOH}$ if needed. The samples were then vortexed, centrifuged, and incubated at $20-22{ }^{\circ} \mathrm{C}$ for $15 \mathrm{~min}$, followed by a $\mathrm{pH}$ readjustment with
$1 \mathrm{M} \mathrm{NaOH}$ to $\sim 9$. The entire procedure was repeated six times for increased labeling efficiency. After the last round of labeling and $\mathrm{pH}$ adjustment, $2.5 \mathrm{M}$ glycine was added, and the samples were incubated for $1 \mathrm{~h}$ to remove excess free labels. A pH adjustment and addition of $2 \mathrm{M}$ hydroxylamine in DMSO followed in order to remove the labels from the tyrosine, serine, and threonine residues of the peptides. After the labeling was complete, the H/D-labeled samples were combined, PepClean C18 spin columns (Pierce, Rockford, IL, USA) were applied to desalt the samples, and the peptides were then eluted using a $70 \%$ aqueous acetonitrile (ACN) solution. Following the removal of the organic solvent in a SpeedVac concentrator (Thermo Scientific, San Jose, CA, USA), the samples (20 $\mu \mathrm{L}$ each) were ready for MS analysis.

The sample preparation procedure described above resulted in four different samples (Samples 1-4) containing various expected ratios of $\mathrm{H} / \mathrm{D}$-labeled peptides (12:1, 8:1, $4: 1,2: 1,1: 1,1: 2,1: 4,1: 8,1: 12$ ), with final peptide concentrations ranging from $250 \mathrm{nM}$ to $300 \mu \mathrm{M}$, producing peptide concentration differences of up to 1200 -fold (see Supplemental Table S1). Each sample was labeled in a forward and a reverse direction: the combination of $\mathrm{H}-\mathrm{SA}$ labeled mixture and D-SA labeled mixture as a forward label, and the combination of D-SA labeled mixture and HSA labeled mixture as a reverse label. In addition, each sample had four labeling replicates, resulting in a total of 32 samples for peptide quantitation on each MS platform (see supplemental Table S1).

\section{MS Analysis}

The three MS platforms used for these comparative peptide quantitation studies were: a UPLC system coupled to an ESI-Q-TOF mass spectrometer (Premier, Waters Corporation, Milford, MA, USA), a capillary LC (Waters) coupled to an ESI-IT mass spectrometer (HCTultra, Bruker Daltonics), and a MALDI-TOF mass spectrometer (UltrafleXtreme, Bruker Daltonics). Whereas the sample mixtures $(1 \mu \mathrm{L}$ of each) were directly injected into the CapLC-ESI-IT instrument, in order to generate a suitable concentration range for the other two platforms, the mixtures needed to be diluted $\sim 30$-fold and 100-fold for the UPLC-ESI-Q-TOF and MALDI-TOF instruments, respectively.

Table 1. The Five Peptide Standards ${ }^{\mathrm{a}}$ Used in this Study

\begin{tabular}{|c|c|c|c|}
\hline Peptide name & Amino acid sequence & Theor. mass (Da) & Theor. masses (H/D) after labeling (Da) \\
\hline Bradykinin 1-7 & RPPGFSP & 756.39 & $856.46 / 860.49$ \\
\hline FVRIamide & NPSSFVRIa & 917.51 & $1017.58 / 1021.61$ \\
\hline Bradykinin & RPPGFSPFR & 1059.56 & $1159.63 / 1163.66$ \\
\hline Angiotensin I & DRVYIHPFHL & 1295.68 & $1395.75 / 1399.78$ \\
\hline Neurotensin & pELYENKPRRPYIL & 1671.90 & $1771.97 / 1776.01$ \\
\hline
\end{tabular}

Theor. mass $=$ theoretical monoisotopic mass; Theor. masses (H/D) after labeling = the expected monoisotopic masses of the H- or D-labeled peptides. The number of SA labels covalently bound to the peptide after labeling is one for all five peptides

a'"p" = N-terminal pyroglutamylation; "a" = C-terminal amidation 
UPLC-ESI-Q-TOF MS The NanoAcquity UPLC system (Waters), equipped with a reversed phase C18 column (Atlantis dC18, $75 \mu \mathrm{m}$ i.d. $\times 15 \mathrm{~cm}, 3 \mu \mathrm{m}$ particle size, and $100 \AA$ pore size, Waters), was used to separate the samples with a mixture of solvent $\mathrm{A}(100 \%$ water with $0.1 \%$ formic acid [FA]) and solvent B (100\% ACN with $0.1 \%$ FA). The 90 min gradient for the UPLC system with a flow rate of $400 \mathrm{~nL} / \mathrm{min}$ was generated as follows: $1 \%$ solvent $\mathrm{B}$ in 0 $30 \mathrm{~min}$ (isocratic); $1 \%-50 \%$ solvent $\mathrm{B}$ in 30-60 min (linear); $50 \%-90 \%$ solvent B in 60-65 min (linear); $90 \%-$ $1 \%$ solvent B in 65-70 min (linear); $1 \%$ solvent B in 70$90 \mathrm{~min}$ (isocratic). Only MS spectra with a scan range of $\mathrm{m} / \mathrm{z}$ 300-2000 were collected.

CapLC-ESI-IT MS Samples were separated on a CapLC system (Micromass, Manchester, UK) with a reversed-phase C18 column (Acclaim PepMap100 C18, $300 \mu \mathrm{m}$ i.d. $\times$ $15 \mathrm{~cm}, 3 \mu \mathrm{m}$ particle size, and $100 \AA$ pore size, Dionex, Sunnyvale, CA, USA). The solvent gradient with a flow rate of $2.5 \mu \mathrm{L} / \mathrm{min}$ was generated by mixing solvent A $(95 \%$ water and $5 \%$ ACN containing $0.1 \%$ FA and $0.01 \%$ trifluoroacetic acid [TFA]) and solvent B (aqueous $95 \%$ ACN containing $0.1 \% \mathrm{FA}$ and $0.01 \% \mathrm{TFA}$ ). The $55 \mathrm{~min}$ gradient for the LC system was created as follows: $3 \%-$ $25 \%$ solvent B in 0-10 min (linear); $25 \%-50 \%$ solvent B in 10-40 min (linear); $50 \%-80 \%$ solvent B in $40-43 \mathrm{~min}$ (linear); $80 \%$ solvent B in 43-45 min (isocratic); $80 \%-3 \%$ solvent B in 45-48 min (linear); $3 \%$ solvent B in 48-55 min (linear). Data were acquired for a MS scan range of $m / z 300$ 2000.

MALDI-TOF MS Samples $(0.5 \mu \mathrm{L})$ were spotted onto a stainless steel MALDI plate (Bruker Daltonics), with $0.5 \mu \mathrm{L}$ of either saturated 2,5-dihydroxybenzoic acid (DHB) or alpha-cyano-4-hydroxycinnamic acid (CHCA) as matrices. Three technical replicates were collected for each spot, with every spectrum comprised of 10 acquisitions of 100 laser shots.

\section{MS Data Analysis}

The identifications of peptide peaks were performed by assessing the combination of charge states, mass matches, and LC retention times. The difference between the $\mathrm{H}$ - and D-labeled peptides with a +1 charge state is $4 \mathrm{Da}$, and with a +2 charge is $2 \mathrm{Da}$. For the data acquired by UPLC-ESI-Q-TOF MS and CapLC-ESI-IT MS, extracted ion chromatograms were examined for both the light-and heavy-labeled peptides, and the mass spectra across the entire elution period for both sets of peaks were summed for quantitation. The intensities of the most abundant isotopic peaks for either the H- or Dlabeled peptides were used to calculate the ratios. For the data acquired by MALDI-TOF MS, after identification of the differentially labeled peptides, the most abundant isotopic peaks were used for ratio calculations.

\section{Results and Discussion}

\section{Experimental Design}

We designed a set of experiments using a variety of peptide mixtures to determine what conditions (e.g., MS platforms, analyte ratios, data acquisition and analysis, etc.) yielded the most accurate relative quantitation of neuropeptides from a mixture. Our sample sets represented variations in neuropeptide amino acid sequences, PTMs, and concentrations. Each sample contained a mixture of five representative neuropeptide standards with masses spread across 500-2000 Da (Table 1), a mass range that is suitable for peptide detection by the three MS platforms. Two out of these five neuropeptides are modified by amidation or pyroglutamylation, two common PTMs present in neuropeptides. These samples also had expected ratios of differentially labeled peptides varying from $12: 1$ to $1: 1$ to $1: 12$, and contained peptide concentrations ranging from $250 \mathrm{nM}$ to $300 \mu \mathrm{M}$. We optimized the samples for two of the MS platforms to ensure the best instrument performance by diluting them 30 -fold $(\sim 8 \mathrm{nM}$ to $10 \mu \mathrm{M})$ and 100 -fold $(2.5 \mathrm{nM}$ to $3 \mu \mathrm{M})$. These mixtures matched an important portion of the range of physiological concentrations of neuropeptides, which spans from micromolar to picomolar [51]. Furthermore, in order to allow for comparisons between different samples, only one of the parameters, either the ratio of differentially labeled peptides or the peptide concentration, was modified when designing these sample mixtures. To account for variations that may result from sample preparation and instrumental fluctuation, four technical replicates were labeled in parallel, and samples were also labeled in both forward and reverse directions. In this way, the potential effects of these various factors on peptide quantitation were addressed.

\section{Peptide Quantitation}

By using SA to label each of the five peptides (supplemental Table S1) in the mixtures, the labeled peptides were $100 \mathrm{Da}$ (H-SA) or $104 \mathrm{Da}$ (D-SA) heavier than the original (unlabeled) peptides (Figure 1). These results indicate that greater than $99 \%$ of the peptides in our mixtures were labeled, regardless of the concentrations of other peptides present in the mixtures; this is because of the more than 1000-fold excess of labeling reagent to peptide (Figure 1). Our results also demonstrate the stability of the labeled peptides during storage as well as during analysis with the three MS platforms we used. This stability is a prerequisite for successful follow-up studies.

The ratios of the peak intensities of the H- or D-labeled peptides in the MS spectra were compared with the theoretical ratios. A $20 \%$ error in the peptide ratios was used as the cutoff, since this value is often used as an accuracy threshold for bioassays, and comparable values have been used in a number of studies to validate isotopic labeling approaches [47, 49, 52]. 


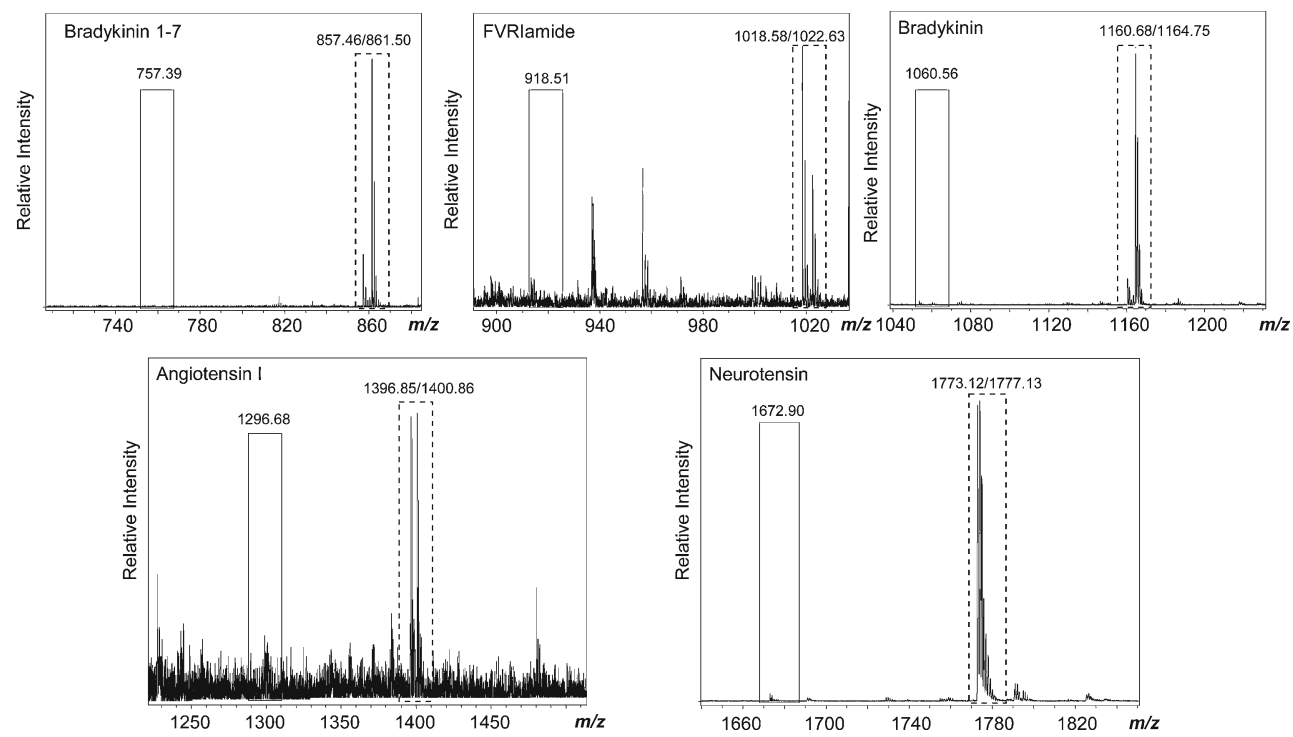

Figure 1. Representative MS spectra of a peptide mixture examined by MALDI-TOF MS indicating complete peptide labeling by $\mathrm{H}$-/D-succinic anhydride (SA). Each spectrum demonstrates the detection of the five standard peptides (bradykinin 1-7, FVRlamide, bradykinin, angiotensin I, and neurotensin) with a +1 charge state. Solid line rectangles indicate the original masses of the peptides before SA labeling showing complete incorporation of the labels. Dashed line rectangles indicate the peaks of H-/D-labeled peptides
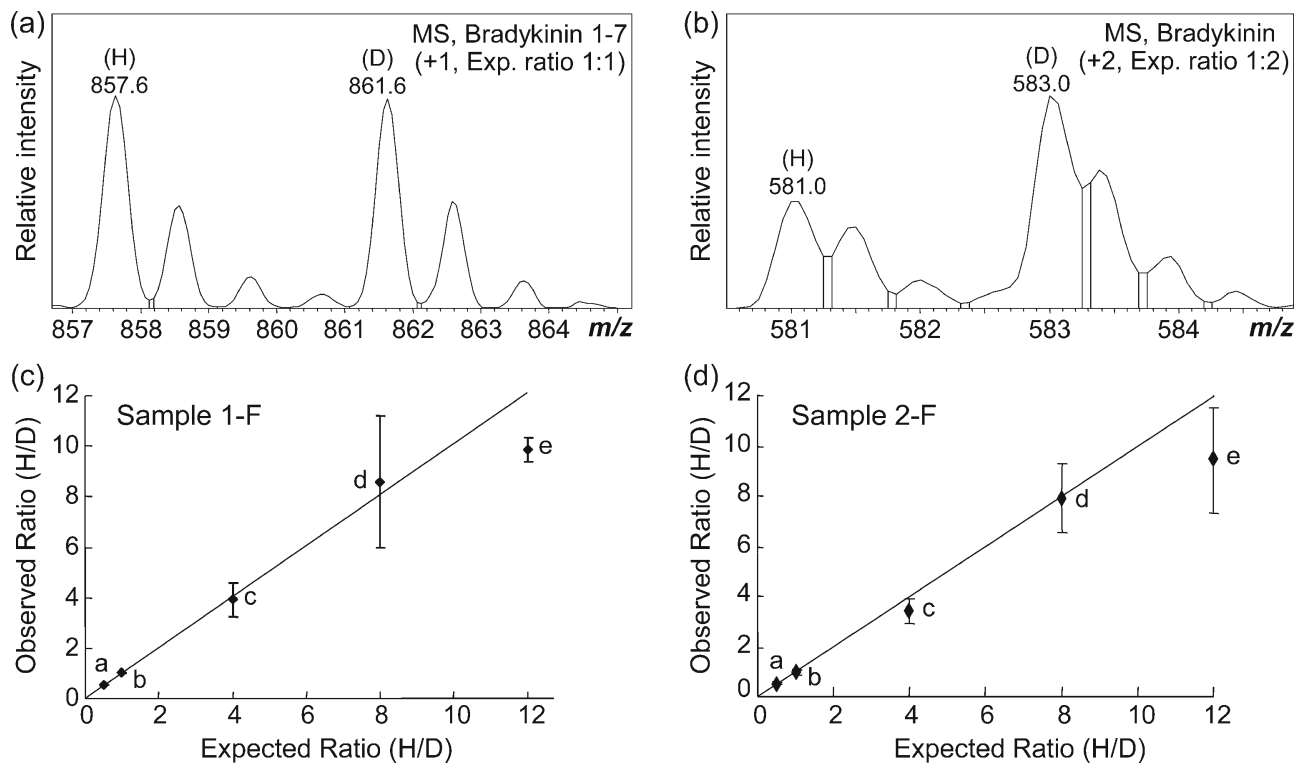

Figure 2. Representative data for quantitation of peptide mixtures by CapLC-ESI-IT MS. (a) MS spectra of the H- and D-forms of bradykinin 1-7 (+1 charge, molecular weight 756.39) with an expected ratio of 1:1 and a calculated ratio of $1.02: 1$ (2 \% error). (b) MS spectra of $\mathrm{H}$ - and D-forms of bradykinin (+1 charge, molecular weight 1059.56) with an expected ratio of $1: 2$ and a calculated ratio of $1: 1.97$ (2 \% error). (c) Sample 1-F with a concentration range of 400 -fold $(0.25 \mu \mathrm{M}$ to $100 \mu \mathrm{M})$ and relative errors of $\leq 20 \%$ for all peptides. (d) Sample $2-F$ with a concentration range of 1200 -fold $(0.25 \mu M$ to $300 \mu M)$ and relative errors of $\leq 20 \%$ for all peptides. Key: a: bradykinin (+2 charge); b: bradykinin 1-7 (+1 charge); c: neurotensin (+2 charge); d: FVRlamide (+1 charge); e: angiotensin I (+2 charge). The Expected Ratio $(H / D)$ is the ratio of $H$ - and D-labeled peptides actually in the mixture, and the Observed Ratio $(H / D)$ is the ratio of the average observed ratios determined from four technical replicates. Error bars are standard deviations; the solid line is the expected 1:1 correlation between observed and expected ratios, and the relative error (\%) is calculated from the average of observed ratios-expected ratio/expected ratio 

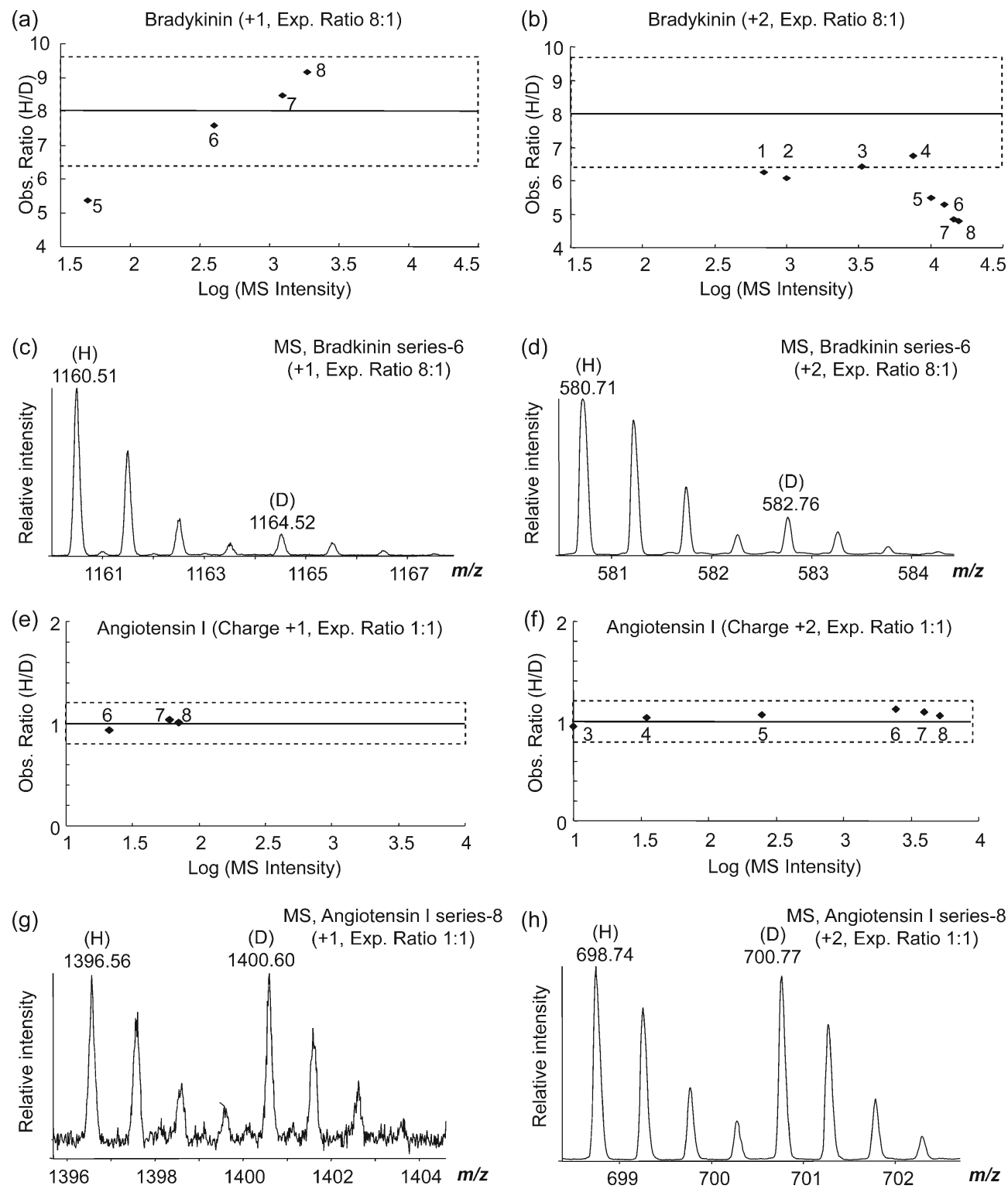

Figure 3. UPLC-ESI-Q-TOF MS quantitative analysis of a serial dilution of Sample 4-R (a concentration range of 1200fold). The order for the amount of sample in Series $1-8$ is $1<2<3<4<5<6<7<8$ (the highest dilution is $~ 10,000$-fold for Series-1). (a), (b) Comparison of the observed ratios of differentially labeled bradykinin standards (expected ratio 8:1) in the serial dilution with (a) +1 , or (b) +2 charge state. (c), (d). A peak pair of differentially labeled bradykinin standards (expected ratio 8:1) in one dilution (Series-6) with (c) +1 charge and observed ratio of $7.59: 1$, or (d) +2 charge and observed ratio of 4.48:1. (e), (f). Comparison of the observed ratios of differentially labeled angiotensin I standards (expected ratio 1:1) in the serial dilution with (e) +1 or (f) +2 charge state. (g), (h). A peak pair of differentially labeled angiotensin I standards (expected ratio 1:1) in one dilution (Series-8) with $(\mathrm{g})+1$ charge and observed ratio of $1.01: 1$, or (h) +2 charge and observed ratio of 1.05:1. Exp. Ratio = the expected ratio of $\mathrm{H}$ - and D-labeled peptides in the mixture; Obs. Ratio $(H / D)=$ the observed ratios of $H$ - and D-labeled peptides in the mixture. The dashed line rectangles indicate that the observed ratio is within $20 \%$ of the expected ratio. The solid lines indicate the expected ratio of $H$ - and $\mathrm{D}$ labeled peptides; the linear region of this MS platform is at best from Log (MS intensity) 2 to 4

Here, whenever the observed ratio of isotopically labeled peptides was within $20 \%$ of the expected ratio, the quantification was considered to be a success.

CapLC-ESI-IT MS One microliter of each of the eight peptide mixtures (with four replicates for each mixture) was injected into the CapLC-ESI-IT MS system. The MS spectra across the entire elution time for all $\mathrm{H}$ - and D-labeled peptides were summed, and the ratios were calculated using their most abundant isotopic peaks. Representative MS spectra of two of the peptides, bradykinin 1-7 and bradykinin, are presented in Figure $2 \mathrm{a}$ and $\mathrm{b}$; the ratios of 
the differentially labeled peptides are within $2 \%$ of the expected ratios of $1: 1$ and 1:2. Representative data of the ratios and concentrations for the five peptides in the two mixtures are shown in Figure $2 \mathrm{c}$ and d, with peptide mixture 1-F having a concentration range from 0.25 to $100 \mu \mathrm{M}$ (400fold range), and peptide mixture $2-\mathrm{F}$ from 0.25 to $300 \mu \mathrm{M}$ (1200-fold range). Samples $1-F$ and $2-F$ have the same expected ratios for the five peptides, but different concentrations. The observed average ratio of each of these differentially labeled peptides was within $20 \%$ of the expected ratio, even for peptides (e.g., angiotensin I) with the highest expected ratio of 12:1 (Figure $2 \mathrm{c}$ and d). Over this range, the peptide levels did not change the observed quantitation accuracy (Figure 2c and d), nor did the accuracy change when changing peptide ratios (data not shown). For peptides with an expected ratio of 1:1, the relative error for the observed ratio was typically less than $10 \%$. Since this platform generated one predominant charge state (either +1 or +2 charge) for each peptide, peptides with only one charge state were quantified here (Figure 2). In summary, the CapLC-ESIIT MS system allowed us to obtain quantitative data for most samples, and the results were consistent between the forward and reverse labeling (data not shown).

UPLC-ESI-Q-TOF MS This platform has been widely employed in the quantitative peptidomics of various biological samples [15, 45, 49], in part because of its high mass resolution and mass accuracy. Nanoscale UPLC-ESI-Q-TOF MS requires lower amounts of samples to be injected than CapLC-ESI-IT MS; therefore, we diluted the samples 30fold. Our initial analysis of these samples demonstrated a narrow linear range of $\sim 50$-fold and some inconsistencies among multiple charge states of the same peptide. In order to understand why, we selected one mixture, sample 4-R, made a serial dilution, and then injected $1 \mu \mathrm{L}$ of eight dilutions into the UPLC-ESI-Q-TOF mass spectrometer to seek ways to improve the quantitation. Our results suggest that if the MS signals for the peptides being measured fall within the linear region of the detector of the UPLC-ESI-Q-TOF instrument (ideally from Log [MS intensity] 2 to 4), the ratios of H/D-labeled peptides are mostly within the $20 \%$ threshold, even for peptides with high ratios (e.g., bradykinin, with an expected ratio of 8:1 in Series 6-8 [+1 charge], and in Series 1-4 [+2 charge]) (Figure $3 a$ and b). On the other hand, if the peptide does not produce an MS signal within the linear range (e.g., due to detector saturation), the differences in peptide levels are distinguished but underestimated (e.g., bradykinin in Series 5-8 [+2 charge]) (Figure 3b). Representative MS spectra of $\mathrm{H}$ - and D-labeled bradykinin $(+1$ and +2 charge states) are shown in Figure $3 \mathrm{c}$ and d. For isotopically labeled peptide pairs with a ratio of $1: 1$, no significant differences were observed, even if the detector was saturated (Figure 3e-h).

Often, ESI generates peptides with multiple charge states, and we commonly observed +1 and +2 charge states. We investigated the measurement accuracy for both charge states. A comparison between Figure 3a and $b$ suggest that the presence of MS signals within the linear region are required for either +1 or +2 charged peptides to be accurately quantified. Interestingly, the MS intensities of peptides with different charge states vary. Often the +2 charge peptide had a much higher MS signal than the +1 charge peptide, with a two-order of magnitude difference observed in many cases (Figure $3 \mathrm{a}, \mathrm{b}$, and e, f). It appears that the peptide with the +2 charge usually saturated the detector before the same peptide with the +1 charge. We took advantage of this observation by selecting ions with the appropriate charge state to quantify, and as a result, the observed ratios for all of the peptides were within $20 \%$ of the expected values. Compared with previous studies showing that ESI-Q-TOF MS has a linear range below 100fold for quantitation $[49,53]$, the UPLC-ESI-Q-TOF MS approach evaluated here successfully quantified peptide mixtures with a concentration range of $>1000$-fold (Figure 4) and peptides in the low femtomole range (Figure 3b, Series-1). (a)

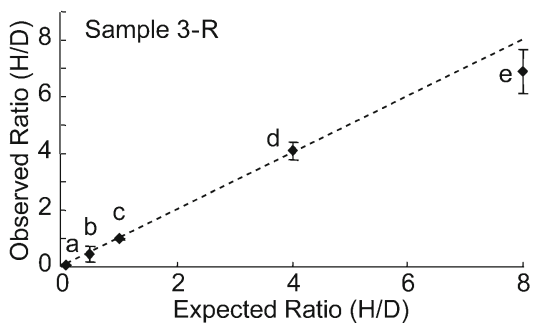

(b)

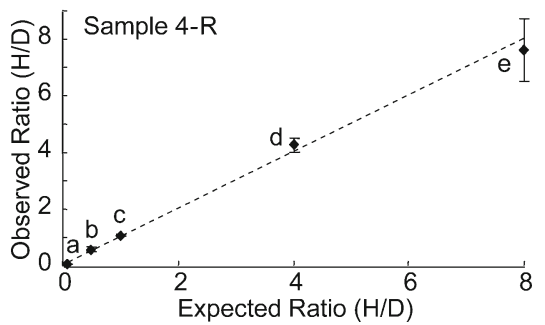

Figure 4. Representative data for quantitation of peptide mixtures by UPLC-ESI-Q-TOF MS. (a) Sample 3-R with a concentration range of 300-fold ( $~ 8 \mathrm{nM}$ to $2.5 \mu \mathrm{M}$ after 30-fold dilution) and a relative error of $\leq 20 \%$. (b) Sample 4-R with a concentration range of 1200 -fold ( $~ 8 \mathrm{nM}$ to $10 \mu \mathrm{M}$ after 30 -fold dilution) and a relative error of $\leq 20 \%$. Key: a: neurotensin; b: FVRlamide; c: angiotensin I; d: bradykinin 1-7; e: bradykinin. The Expected Ratio $(H / D)=$ the ratio of the H- and D-labeled peptides actually in the mixture and the Observed Ratio $(H / D)=$ the average observed ratio determined from four technical replicates. Error bars are standard deviations; the dashed lines are the expected 1:1 correlation between the observed and expected ratios, and the relative error (\%) is calculated from the average of observed ratios-expected ratio/expected ratio 
Table 2. Representative Data for Quantitation of Peptide Mixtures by MALDI-TOF MS Using DHB as Matrix ${ }^{\mathrm{a}}$

\begin{tabular}{|c|c|c|c|c|}
\hline Peptide name & Final conc. of $\mathrm{H} / \mathrm{D}$ after dilution (pM) & Exp. ratio of $\mathrm{H} / \mathrm{D}$ & Avg. ratio of $\mathrm{H} / \mathrm{D} \pm \mathrm{SD}$ & Error $(\%)$ \\
\hline \multicolumn{5}{|c|}{ Sample 1-F (concentration range of 400 -fold) } \\
\hline Bradykinin 1-7 & $125 / 125$ & 1 & $1.09 \pm 0.05$ & 9 \\
\hline FVRIamide & $20 / 2.5$ & 8 & $16.52 \pm 0.32$ & 106 \\
\hline Bradykinin & $62.5 / 125$ & 0.5 & $0.53 \pm 0.02$ & 6 \\
\hline Angiotensin I & $150 / 12.5$ & 12 & $21.10 \pm 1.37$ & 76 \\
\hline Neurotensin & $1000 / 250$ & 4 & $3.72 \pm 0.48$ & -7 \\
\hline \multicolumn{5}{|c|}{ Sample $2-\mathrm{F}$ (concentration range of 1200 -fold) } \\
\hline Bradykinin 1-7 & $12.5 / 12.5$ & 1 & $0.84 \pm 0.03$ & -16 \\
\hline FVRIamide & $1000 / 125$ & 8 & $8.49 \pm 1.13$ & 6 \\
\hline Bradykinin & $2.5 / 5$ & 0.5 & N/A & N/A \\
\hline Angiotensin I & $3000 / 250$ & 12 & $10.50 \pm 0.63$ & -13 \\
\hline Neurotensin & $250 / 62.5$ & 4 & $6.08 \pm 0.26$ & 52 \\
\hline \multicolumn{5}{|c|}{ Sample 4-R (concentration range of 1200 -fold) } \\
\hline Bradykinin 1-7 & $500 / 125$ & 4 & $5.00 \pm 1.24$ & 25 \\
\hline FVRIamide & $2.5 / 5$ & 0.5 & $0.47 \pm 0.04$ & -6 \\
\hline Bradykinin & $500 / 62.5$ & 8 & $9.42 \pm 0.92$ & 18 \\
\hline Angiotensin I & $12.5 / 12.5$ & 1 & $1.05 \pm 0.12$ & 6 \\
\hline Neurotensin & $250 / 3000$ & 0.083 & $0.095 \pm 0.01$ & 13.5 \\
\hline
\end{tabular}

${ }^{\mathrm{a}}$ Final conc. of $\mathrm{H} / \mathrm{D}=$ the final concentrations for both $\mathrm{H}$ - and D-labeled peptides in the mixture; exp. ratio of $\mathrm{H} / \mathrm{D}=$ the Expected ratio of $\mathrm{H}$ - and $\mathrm{D}-\mathrm{labeled}$ peptides in the mixture; Avg. ratio of $\mathrm{H} / \mathrm{D}=$ the average ratios of four technical replicates of a peptide in the mixtures; $\mathrm{SD}=$ standard deviation; error (\%) is calculated from the average ratio-expected ratio/expected ratio, numbers in bold are within $20 \%$ of the expected ratios, and the negative sign indicates that the average ratio is lower than the expected ratio. Samples were diluted 100-fold for the MALDI-TOF MS studies

MALDI-TOF MS One key advantage of MALDI-TOF MS, compared with other MS platforms, is that it requires much lower sample amounts for analysis and can detect peptides at attomole levels. Therefore, we diluted our samples 100 -fold for MALDI-TOF MS, spotted $0.5 \mu \mathrm{L}$ of the diluted samples with either DHB or CHCA as matrices on the MALDI target, and made a comparison among the samples. Ten acquisitions of MS signals were obtained uniformly across a single sample spot and added together for quantitation purposes.

Similar to previous reports $[47,54]$, our initial results suggested that without any optimization, MALDI analysis is able to differentiate peptide levels, even for the lowest level peptides, but may only provide accurate quantitation for peptides with a concentration difference of about 20 -fold or less (data not shown). However, by selecting the appropriate data acquisition protocols, we were able to improve the effective dynamic range of the MALDI-TOF instrument. First, we made sure that the higher abundance peptides did not saturate the detector. Saturation of the MALDI-TOF detector is easily identified, and often can be simply fixed by modifying the parameters of the mass spectrometer (e.g., laser power). In addition, we required the peak intensity of peptides to be above a certain signal-to-noise threshold, and optimized the acquisition parameters to make sure that the lower concentration peaks met this threshold requirement, even for the peptides at the lowest abundance.

The data obtained with DHB as the matrix, and the optimized acquisition parameters, are shown in Table 2. For most of the peptides, the observed ratios were within $20 \%$ of the theoretical ratios. However, the dynamic range for MALDI-TOF MS is sample-dependent. For Sample 4-R, MALDI-TOF MS provided accurate quantification of peptides over a range of $\sim 1200$-fold, from 2.5 to $3000 \mathrm{pM}$. With
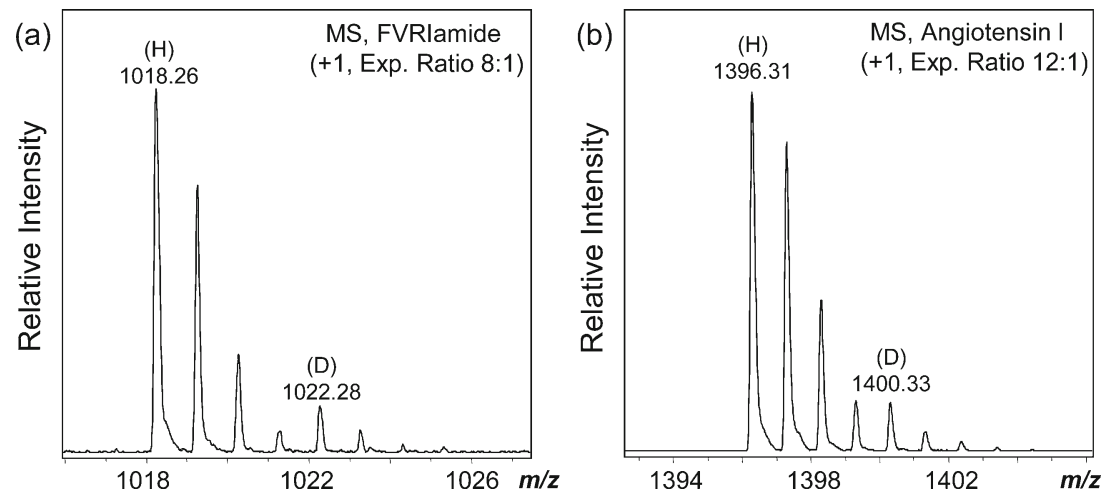

Figure 5. MS spectra of Sample 1-F for peptide quantitation via a single acquisition by MALDI-TOF MS (a) H- and D-forms of FVRlamide (+1 charge, molecular weight 917.51) with an expected ratio of 8:1 and calculated of ratio $8.5: 1$ (7 \% error). (b) $\mathrm{H}$ and D-forms of angiotensin I (+1 charge, molecular weight 1295.68) with an expected ratio of $12: 1$ and a calculated ratio of 12.4:1 (3 \% error) 
Table 3. Representative Data for Quantitation of Peptide Mixtures Using CHCA Versus DHB as Matrices by MALDI-TOF MS ${ }^{\text {a }}$

\begin{tabular}{lccc}
\hline Peptide name & Final conc. of H/D after dilution (pM) & Exp. ratio of H/D & Avg. ratio of H/D \pm SD \\
\hline Sample 4-R with CHCA (concentration range of 1200-fold) & & \multicolumn{2}{c}{ Error (\%) } \\
Bradykinin 1-7 & $500 / 125$ & 4 & $6.19 \pm 0.36$ \\
FVRIamide & $2.5 / 5$ & 0.5 & $0.40 \pm 0.03$ \\
Bradykinin & $500 / 62.5$ & 8 & $11.83 \pm 1.20$ \\
Angiotensin I & $12.5 / 12.5$ & 1 & $0.92 \pm 0.07$ \\
Neurotensin & $250 / 3000$ & 0.083 & $0.10 \pm 0.01$ \\
Sample 4-R with DHB (concentration range of 1200-fold) & $500 / 125$ & 4 & $5.00 \pm 1.24$ \\
Bradykinin 1-7 & $2.5 / 5$ & 0.5 & $0.47 \pm 0.04$ \\
FVRIamide & $500 / 62.5$ & 8 & $9.42 \pm 0.92$ \\
Bradykinin & $12.5 / 12.5$ & 1 & $1.05 \pm 0.12$ \\
Angiotensin I & $250 / 3000$ & 0.083 & $0.095 \pm 0.01$ \\
Neurotensin & & $-\mathbf{8}$ \\
\hline
\end{tabular}

${ }^{\mathrm{a}}$ Final conc. of $\mathrm{H} / \mathrm{D}=$ the final concentrations for both $\mathrm{H}-$ and D-labeled peptides in the mixture; exp. ratio of $\mathrm{H} / \mathrm{D}=$ the expected ratio of $\mathrm{H}-$ and $\mathrm{D}-\mathrm{labeled}$ peptides in the mixture; avg. ratio of $\mathrm{H} / \mathrm{D}=$ the average ratios of four technical replicates of a peptide in the mixtures; $\mathrm{SD}=$ standard deviation; error $(\%)$ is calculated from the average ratio-expected ratio/expected ratio, numbers in bold are within $20 \%$ of the expected ratios, and the minus sign indicates that the average ratio is lower than the expected ratio; samples here were diluted 100-fold for the MALDI-TOF MS studies

Sample 2-F and the same concentration range, peptides could be accurately quantified over a range of $\sim 240$-fold, from 12.5 to $3000 \mathrm{pM}$. But for Sample 1-F, accurate quantitation of peptides could only be obtained over a range of $\sim 20$ fold, from 62.5 to $1000 \mathrm{pM}$.

Notably, two types of peptides were harder to quantify by MALDI-TOF MS. One type is peptide pairs having a high expected ratio (Table 2, FVRIamide and angiotensin I in Sample 1-F), and the other is low abundance peptides that generate low MS signals (Table 2, bradykinin and neurotensin in Sample 2-F). When this situation was combined, we generally observed a larger ratio of the differentially labeled peptides than the expected ratio (Table 2, FVRIamide and angiotensin I in Sample 1-F, and neurotensin in Sample 2-F). This may be due to the more prominent effects of ion suppression of one labeled peptide by another. However, the observed ratio still correctly reflected the actual 1:1 ratio, even if the peptide was at a low level (Table 2, angiotensin I in Sample 4-R, and bradykinin 1-7 in Sample 2-F).

It is also worth mentioning that if we want to obtain relatively accurate quantitation of one specific peptide in low abundance, instrumental parameters can be adjusted or optimized just for that peptide of interest; using these approaches we can acquire MS signals above a threshold, while taking care not to saturate the detector. One single MS acquisition, instead of several summed spectra across the sample, is enough to achieve good quantitation accuracy. As shown in Figure 5, the accuracy for low abundance FVRIamide and angiotensin I are $7 \%$ and $3 \%$, respectively, after optimization and single MS acquisition, whereas previous results without such adjustments reported larger errors, $106 \%$ and $76 \%$ respectively (Table 2, Sample 1-F). These results could only be obtained for peptides above the low femtomole level, the limit of quantification (LOQ) for our MALDI-TOF MS platform.

It is also important to ask, what are the effects of the MALDI matrix? Besides DHB, we tested the other common matrix, CHCA. As shown in Table 3, the use of DHB led to an accurate quantitation of most peptides in Sample 4-R over a concentration range of $\sim 1200$-fold, from 2.5 to $3000 \mathrm{pM}$; in contrast, CHCA was less robust and the percentage of error was higher for all five peptides examined using this matrix as compared to DHB. Because CHCA is reported to provide a more consistent MS signal across the sample spot [54], we expected it to provide better quantitation performance. It is possible that the differentially labeled peptides cocrystallized differently in DHB than CHCA. More likely, perhaps DHB and its "sweet spots" facilitate the enrichment of peptides into a small region. This would lead to a more intense MS signal, making it easier to meet threshold requirements, and thus provide a wider concentration range and a lower LOQ. For most peptides, low femtomole or even lower levels of peptides are required when using DHB as a matrix for accurate quantitation, consistent with previous studies [20]. Although a MALDI-TOF mass spectrometer is not commonly considered to be the ideal instrument for peptide quantitation [54], our results suggest that MALDI-TOF MS can differentiate changes in peptide levels.

\section{Conclusions}

Using an isotopic labeling approach, we compared the ability of three different MS platforms to accurately quantitate neuropeptides in mixtures having concentration ranges greater than three orders of magnitude and various complexities. The three MS platforms performed differently, with unique performance specifications and working concentration ranges. The CapLC-ESI-IT system handled a wide dynamic range of peptides with an LOQ of $\sim 100 \mathrm{fmol}$. By selecting the appropriate charge state to quantify, the dynamic range of accurate quantitation using UPLC-ESI-QTOF MS has been improved by at least one order of magnitude over previously reported results [49, 53]. Prior studies have demonstrated that MALDI-TOF MS can provide quantitative results for peptides with relatively narrow concentration ranges (perhaps less than two orders 
of magnitude [47, 54]). Here, by optimizing the acquisition parameters, the ability of MALDI MS to allow us to quantify peptides was improved to greater than 1200-fold (although this was sample-dependent). Despite having the lowest LOQ among the three platforms, the MALDI-TOF instrument provided simpler data analysis. The approaches outlined here led to a 10-fold larger dynamic range than previously reported for isotopic labeling approaches [36, 46, 47, 49]. It is easier to quantify peptides close to the $1: 1$ ratio, but such ideal situations are not always encountered in practical neuropeptide measurements; therefore, understanding the limits of the strategies being used to measure peptides is important. Although the current study is limited to samples composed of five neuropeptide standards, most tissue/brain samples contain hundreds to thousands of neuropeptides, which may make quantitation more complex than the scenarios presented here. While additional chromatographic separation and other clean-up steps may be necessary for real-world tissue samples, the approaches developed using the well-defined samples assayed here should aid in achieving such measurements. Clearly, the MS platforms evaluated in this study are valuable and generalizable, but we must keep in mind that instrument specifications will inevitably change as the technology evolves, and thus, will require ongoing validation.

\section{Acknowledgments}

The authors thank Dr. Peter Yau and Dr. Brian S. Imai at the Biotechnology Center of UIUC for assistance with UPLCESI-Q-TOF MS. The work was supported by the National Institute on Drug Abuse under Award no. P30 DA018310 and the National Institute of Neurological Disease and Stroke under Award no. R01 NS031609. The content is solely the responsibility of the authors and does not necessarily represent the official views of the award agencies.

\section{References}

1. Fricker, L.D.: Neuropeptide-processing enzymes: applications for drug discovery. AAPS J. 7, E449-E455 (2005)

2. Hook, V., Funkelstein, L., Lu, D., Bark, S., Wegrzyn, J., Hwang, S.R.: Proteases for processing proneuropeptides into peptide neurotransmitters and hormones. Annu. Rev. Pharmacol. Toxicol. 48, 393-423 (2008)

3. Li, L., Sweedler, J.V.: Peptides in the brain: mass spectrometry-based measurement approaches and challenges. Annu. Rev. Anal. Chem. 1, 451-83 (2008)

4. Dhanvantari, S., Seidah, N.G., Brubaker, P.L.: Role of prohormone convertases in the tissue-specific processing of proglucagon. Mol. Endocrinol. 10, 342-355 (1996)

5. Rouille, Y., Bianchi, M., Irminger, J.C., Halban, P.A.: Role of the prohormone convertase $\mathrm{PC} 2$ in the processing of proglucagon to glucagon. FEBS Lett. 413, 119-123 (1997)

6. Rouille, Y., Kantengwa, S., Irminger, J.C., Halban, P.A.: Role of the prohormone convertase $\mathrm{PC} 3$ in the processing of proglucagon to glucagon-like peptide 1. J. Biol. Chem. 272, 32810-32816 (1997)

7. Zheng, M., Streck, R.D., Scott, R.E., Seidah, N.G., Pintar, J.E.: The developmental expression in rat of proteases furin, PC1, PC2, and carboxypeptidase E: implications for early maturation of proteolytic processing capacity. J. Neurosci. 14, 4656-4673 (1994)
8. Baggerman, G., Verleyen, P., Clynen, E., Huybrechts, J., De Loof, A., Schoofs, L.: Peptidomics. J. Chromatogr. B Analyt. Technol. Biomed. Life Sci. 803, 3-16 (2004)

9. Hummon, A.B., Amare, A., Sweedler, J.V.: Discovering new invertebrate neuropeptides using mass spectrometry. Mass Spectrom. Rev. 25, 77-98 (2006)

10. Svensson, M., Skold, K., Svenningsson, P., Andren, P.E.: Peptidomicsbased discovery of novel neuropeptides. J. Proteome Res. 2, 213219 (2003)

11. Fricker, L.D., Lim, J., Pan, H., Che, F.Y.: Peptidomics: identification and quantification of endogenous peptides in neuroendocrine tissues. Mass Spectrom. Rev. 25, 327-344 (2006)

12. Annangudi, S.P., Luszpak, A.E., Kim, S.H., Ren, S., Hatcher, N.G., Weiler, I.J., Thornley, K.T., Kile, B.M., Wightman, R.M., Greenough, W.T., Sweedler, J.V.: Neuropeptide release is impaired in a mouse model of fragile X mental retardation syndrome. ACS Chem. Neurosci. 1, 306-314 (2010)

13. Hatcher, N.G., Atkins Jr., N., Annangudi, S.P., Forbes, A.J., Kelleher, N.L., Gillette, M.U., Sweedler, J.V.: Mass spectrometry-based discovery of circadian peptides. Proc. Natl. Acad. Sci. U. S. A. 105, 12527$12532(2008)$

14. Hatcher, N.G., Sweedler, J.V.: Aplysia bag cells function as a distributed neurosecretory network. J. Neurophysiol. 99, 333-343 (2008)

15. Brockmann, A., Annangudi, S.P., Richmond, T.A., Ament, S.A., Xie, F., Southey, B.R., Rodriguez-Zas, S.R., Robinson, G.E., Sweedler, J.V.: Quantitative peptidomics reveal brain peptide signatures of behavior. Proc. Natl. Acad. Sci. U. S. A. 106, 2383-2388 (2009)

16. Che, F.Y., Vathy, I., Fricker, L.D.: Quantitative peptidomics in mice: effect of cocaine treatment. J. Mol. Neurosci. 28, 265-275 (2006)

17. Che, F.Y., Yuan, Q., Kalinina, E., Fricker, L.D.: Peptidomics of Cpe fat/fat mouse hypothalamus: effect of food deprivation and exercise on peptide levels. J. Biol. Chem. 280, 4451-4461 (2005)

18. Collins, J.J.I.I.I., Hou, X., Romanova, E.V., Lambrus, B.G., Miller, C.M., Saberi, A., Sweedler, J.V., Newmark, P.A.: Genome-wide analyses reveal a role for peptide hormones in planarian germline development. PLoS Biol. 8, e1000509 (2010)

19. Decaillot, F.M., Che, F.Y., Fricker, L.D., Devi, L.A.: Peptidomics of Cpefat/fat mouse hypothalamus and striatum: effect of chronic morphine administration. J. Mol. Neurosci. 28, 277-284 (2006)

20. Rubakhin, S.S., Sweedler, J.V.: Quantitative measurements of cell-cell signaling peptides with single-cell MALDI MS. Anal. Chem. 80, 7128$7136(2008)$

21. Wardman, J.H., Zhang, X., Gagnon, S., Castro, L.M., Zhu, X., Steiner, D.F., Day, R., Fricker, L.D.: Analysis of peptides in prohormone convertase $1 / 3$ null mouse brain using quantitative peptidomics. $J$. Neurochem. 114, 215-225 (2010)

22. Higgs, R.E., Knierman, M.D., Gelfanova, V., Butler, J.P., Hale, J.E.: Comprehensive label-free method for the relative quantification of proteins from biological samples. J. Proteome Res. 4, 1442-1450 (2005)

23. Hixson, K.K.: Label-free relative quantitation of prokaryotic proteomes using the accurate mass and time tag approach. Methods Mol. Biol. 492, 39-63 (2009)

24. Jin, S., Daly, D.S., Springer, D.L., Miller, J.H.: The effects of shared peptides on protein quantitation in label-free proteomics by LC/MS/MS. J. Proteome Res. 7, 164-169 (2008)

25. Wiener, M.C., Sachs, J.R., Deyanova, E.G., Yates, N.A.: Differential mass spectrometry: a label-free LC-MS method for finding significant differences in complex peptide and protein mixtures. Anal. Chem. 76, 6085-6096 (2004)

26. Wickramasekara, S., Neilson, J., Patel, N., Breci, L., Hilderbrand, A., Maier, R.M., Wysocki, V.: Proteomics analyses of the opportunistic pathogen Burkholderia vietnamiensis using protein fractionations and mass spectrometry. J. Biomed. Biotechnol. Article ID 701928, 10 pages (2011)

27. Zhu, W., Smith, J.W., Huang, C.M.: Mass spectrometry-based labelfree quantitative proteomics. J. Biomed. Biotechnol. Article ID 840518, 6 pages $(2010)$

28. Mueller, L.N., Brusniak, M.Y., Mani, D.R., Aebersold, R.: An assessment of software solutions for the analysis of mass spectrometry based quantitative proteomics data. J. Proteome Res. 7, 51-61 (2008)

29. Old, W.M., Meyer-Arendt, K., Aveline-Wolf, L., Pierce, K.G., Mendoza, A., Sevinsky, J.R., Resing, K.A., Ahn, N.G.: Comparison of label-free methods for quantifying human proteins by shotgun proteomics. Mol. Cell. Proteomics 4, 1487-1502 (2005) 
30. Gobom, J., Kraeuter, K.O., Persson, R., Steen, H., Roepstorff, P., Ekman, R.: Detection and quantification of neurotensin in human brain tissue by matrix-assisted laser desorption/ionization time-of-flight mass spectrometry. Anal. Chem. 72, 3320-3326 (2000)

31. Desiderio, D.M.: Mass spectrometric quantification of neuropeptides. Methods Mol. Biol. 61, 57-65 (1996)

32. Dass, C., Kusmierz, J.J., Desiderio, D.M.: Mass spectrometric quantification of endogenous beta-endorphin. Biol. Mass Spectrom. 20, 130-138 (1991)

33. Kusmierz, J.J., Sumrada, R., Desiderio, D.M.: Fast atom bombardment mass spectrometric quantitative analysis of methionine-enkephalin in human pituitary tissues. Anal. Chem. 62, 2395-2400 (1990)

34. Jo, K., Heien, M.L., Thompson, L.B., Zhong, M., Nuzzo, R.G., Sweedler, J.V.: Mass spectrometric imaging of peptide release from neuronal cells within microfluidic devices. Lab Chip 7, 1454-1460 (2007)

35. Zhong, M., Lee, C.Y., Croushore, C.A., Sweedler, J.V.: Label-free quantitation of peptide release from neurons in a microfluidic device with mass spectrometry imaging. Lab Chip 12, 2037-2045 (2012)

36. Canas, B., Lopez-Ferrer, D., Ramos-Fernandez, A., Camafeita, E., Calvo, E.: Mass spectrometry technologies for proteomics. Brief Funct. Genomics Proteomics 4, 295-320 (2006)

37. Bantscheff, M., Schirle, M., Sweetman, G., Rick, J., Kuster, B.: Quantitative mass spectrometry in proteomics: a critical review. Anal. Bioanal. Chem. 389, 1017-1031 (2007)

38. Ji, J., Chakraborty, A., Geng, M., Zhang, X., Amini, A., Bina, M., Regnier, F.: Strategy for qualitative and quantitative analysis in proteomics based on signature peptides. J. Chromatogr. B Biomed. Sci. Appl. 745, 197-210 (2000)

39. Zhang, R., Sioma, C.S., Wang, S., Regnier, F.E.: Fractionation of isotopically labeled peptides in quantitative proteomics. Anal. Chem. 73, 5142-5149 (2001)

40. Che, F.Y., Biswas, R., Fricker, L.D.: Relative quantitation of peptides in wild-type and Cpe(fat/fat) mouse pituitary using stable isotopic tags and mass spectrometry. J. Mass Spectrom. 40, 227-237 (2005)

41. Che, F.Y., Eipper, B.A., Mains, R.E., Fricker, L.D.: Quantitative peptidomics of pituitary glands from mice deficient in copper transport. Cell. Mol. Biol. (Noisy-le-Grand) 49, 713-722 (2003)

42. Xuereb, F., Chaignepain, S., Breilh, D., Godde, F., Saux, M.C., Lenz, C., Glueckmann, M., Schmitter, J.M.: Quantitative analysis of erythropoietin in human plasma by tandem mass spectrometry. Anal. Bioanal. Chem. 400, 2073-2084 (2011)
43. Bark, S.J., Lu, W.D., Hook, V.: Linear and accurate quantitation of proenkephalin-derived peptides by isotopic labeling with internal standards and mass spectrometry. Anal. Biochem. 389, 18-26 (2009)

44. Che, F.Y., Fricker, L.D.: Quantitation of neuropeptides in Cpe(fat)/Cpe (fat) mice using differential isotopic tags and mass spectrometry. Anal. Chem. 74, 3190-3198 (2002)

45. Che, F.Y., Fricker, L.D.: Quantitative peptidomics of mouse pituitary: comparison of different stable isotopic tags. J. Mass Spectrom. 40, 238249 (2005)

46. Choi, Y.S., Pennington, C.L., Wood, T.D.: Stable isotope labeling method targeting terminal tyrosine for relative peptide quantitation using mass spectrometry. Anal. Biochem. 401, 15-21 (2010)

47. Hsu, J.L., Huang, S.Y., Chow, N.H., Chen, S.H.: Stable-isotope dimethyl labeling for quantitative proteomics. Anal. Chem. 75, 6843$6852(2003)$

48. Wang, J., Zhang, Y., Xiang, F., Zhang, Z., Li, L.: Combining capillary electrophoresis matrix-assisted laser desorption/ionization mass spectrometry and stable isotopic labeling techniques for comparative crustacean peptidomics. J. Chromatogr. A 1217, 4463-4470 (2010)

49. Angel, P.M., Orlando, R.: Quantitative carbamylation as a stable isotopic labeling method for comparative proteomics. Rapid Commun. Mass Spectrom. 21, 1623-1634 (2007)

50. DeKeyser, S.S., Li, L.: Matrix-assisted laser desorption/ionization Fourier transform mass spectrometry quantitation via in cell combination. Analyst 131, 281-290 (2006)

51. D’haenen, H., den Boer, J.A., Willner, P.: Biological Psychiatry, 2nd ed. John Wiley and Sons, 757 vol. 1, p 1486 (2002)

52. Blagojevic, V., Zhidkov, N., Tharmaratnam, S., Pham, V.T., Kaplan, H., Bohme, D.K.: Peptide quantitation with methyl iodide isotopic tags and mass spectrometry. Analyst 135, 1456-1460 (2010)

53. Quintana, L.F., Campistol, J.M., Alcolea, M.P., Banon-Maneus, E., SolGonzalez, A., Cutillas, P.R.: Application of label-free quantitative peptidomics for the identification of urinary biomarkers of kidney chronic allograft dysfunction. Mol. Cell. Proteomics 8, 1658-1673 (2009)

54. Szajli, E., Feher, T., Medzihradszky, K.F.: Investigating the quantitative nature of MALDI-TOF MS. Mol. Cell. Proteomics 7, 2410-2418 (2008) 